\title{
Ethical Discussion and Approach for the Disabling Acute Subdural Hematoma
}

\author{
Calvin W. Howard BSc (Hon,), MD \\ Cumming School of Medicine, University of Calgary, Alberta, Canada. \\ E-mail: calvin.howard@ucalgary.ca \\ ORCID: 0000-0001-5576-9608
}

\begin{abstract}
:
Neurosurgical patients face dire outcomes infringing upon their functional capacity. Often, the impairment of a neurosurgical patient may be so severe patients will indefinitely require assistance with the majority of activities of daily living. These patients may be left unable to communicate, be left largely unable to interact with their environment, and may very well be unable to comprehend the world around them to their previous extent. Over decades of exposure to such tragic outcomes, it is not uncommon for neurosurgeons to ponder if these patients would have been better off dead. Within the common situation of a severe acute subdural hematoma, there is often controversy amongst the surgical team as to whether an operation should occur as the patient is often left severely impaired at best. To further explore the ethics of when to operate, the case of a severe acute subdural hematoma is used to guide discussion and ultimately suggest approaches in practice.
\end{abstract}

\section{INTRODUCTION}

Neurosurgery has long enamored mankind with its fundamental impact upon humanity. To operate on the anatomical substrate of the self poses common dilemmas. Treatment of a neurosurgical condition commonly results in permanent neurological deficit. ${ }^{1}$ In extreme cases, preservation of life may result in irreparable loss of function and self. ${ }^{2}$ Furthermore, outcomes are often difficult to predict, and patients may range from moderately impaired to dead in severe neurotraumas. ${ }^{2}$ It is undeniable that severe neurotrauma commonly results in impairment precluding return to social activities or work, even causing complete dependence in activities of daily living.

To navigate such difficulties, medical ethics employs the four tenets of justice, autonomy, beneficence, and nonmaleficence. However, within the context of neurosurgery, balancing these tenets becomes difficult, especially regarding beneficence and non-maleficence. Neurosurgeons and allied healthcare have lamented more harm is done by saving life in severe pathologies. ${ }^{2-4}$ Some have referred to these post-surgical states as "sub-human", and debate continues regarding worth of surgery. ${ }^{3-6}$ This debate focuses on surgery making quality of life unacceptably low in the context of permanent severe functional impairment.

Occurring in $10-20 \%$ of mild traumatic brain injury, ${ }^{7-9}$ and up to $60 \%$ of severe traumatic brain injury, acute subdural hematomas are a common neurosurgical pathology. ${ }^{10}$ Moreover, ethics of when and how to operate upon this ubiquitous presentation remains largely undetermined. ${ }^{2,4}$ In an attempt to provide an answer, the ethics of operating in the case of the severe acute subdural hematoma and a practical approach are explored. Specifically, the ethical ability to appraise post-operative quality of life and the impact of this appraisal upon decision to operate are discussed. 


\section{CASE PRESENTATION}

A 55 year old woman presents to the trauma bay with altered level of consciousness after a reported fall seven hours earlier.

In the trauma bay, the flight medic explains emergency medical services were contacted when she had been found confusedly wandering a local grocery store of her rural town. Upon arrival, the woman was obtunded and brought to the town's emergency department. No further identifying information could be found and she was unable to provide emergency contact information. Neurological examination revealed equal and reactive pupils, eyes opening to pain, no vocalization, and extensor posturing, providing a Glasgow Coma Score (GCS) of 5. The patient was intubated and a CT scan demonstrated an acute left subdural hematoma measuring 4 millimetres with 3 millimetres of midline shift.

The patient was transferred by air ambulance to the nearest neurosurgical care centre. Upon arrival, her head was elevated, blood pressure controlled, mild hypocarbia maintained, mannitol provided, and a repeat CT demonstrated a right frontoparietal acute subdural hematoma of 13 millimetres and 7 millimetres of midline shift with right uncal herniation.

Upon return from the CT scan, examination revealed an unreactive 8 millimetre right pupil, a reactive 5 millimetre left pupil, no eye opening, no verbalization, no motor activity in the left limbs and extensor posturing in the right limbs, providing a GCS of 3. At this point, the patient is still unable to be identified, no contacts are known, and no further information can be provided.

If the hematoma is not immediately evacuated, she will die. If the hematoma is evacuated, she may still die, may be left ventilator-dependent, or may be left permanently dependent with moderate to severe functional impairment. Given the severity of her initial brain injury, it is most likely she will have permanent severe neurological deficit if she survives.

\section{CASE DISCUSSION}

Although not applicable to the case above, it is common for patients to be identifiable. Lengthy discussion with substitute decision makers is then necessary in deciding to operate. In the age of patient autonomy and decreased paternal nature of medicine, more emphasis is being placed upon substitute decision maker opinions. However, substitute decision makers may be easily swayed given the significant power differential between them and neurosurgeons. This causes bias or subtle coercion, undermining autonomy and ethical decision making. Commonly, the discussion centers around a patient's predicted quality of life post-surgery. For example, a younger patient may be portrayed as having a reasonable chance of achieving some meaningful life, while an older patient may be presented as being forced into a poor quality of life by the operation. Situations are often preferentially represented based upon the neurosurgeon's beliefs. With or without a substitute decision maker, the neurosurgeon's threshold for surgery is largely determined by presumed patient quality of life. To address management decisions both with and without substitute decision makers, the neurosurgeon's approach must be analyzed independently. This illustrative case presents a situation in which the neurosurgeon stands as the sole or predominant decision maker, allowing isolated assessment of neurosurgical ethics.

The severely impaired functional capacity after acute subdural hematoma has been described as a "sub-human state". ${ }^{5}$ Although operation is undoubtedly life-saving, the significant functional morbidity has led the neurosurgical community to question the worth of operation in severe cases. ${ }^{2,11}$ It is not uncommon for neurosurgeons to bemoan these people as "better off dead". 4,5 Commonly, members of the general populace mention they would in fact rather be dead than live in such an impaired state, finding it highly undesirable. ${ }^{3}$ These perspectives indicate to live severely functionally impaired is not worth living.

To explore the previously mentioned opinions, consider two equally impaired patients. A neurosurgical patient left 
dependent upon others for activities of daily living and an equally dependent congenitally impaired patient. If a functionally impaired life is not worth living, we would consider the congenitally impaired patient "better off dead" too- an unethical notion consistent with eugenics. More accurately, people are bemoaned "better off dead" due to loss of function relative to their baselines. If relative functional impairment were the critical factor in determining worth of life, it would be reasonable to assume patients with acquired severe impairment would report life as not worth living. However, functionally impaired patients often report their quality of life and desire to live as quite high. Conversely, healthcare workers perceive the same patient's quality of life as low, a concept known as the disability paradox. ${ }^{12,13}$ This demonstrates a change in perspective those with acquired impairment may undergo as well as bias developed by healthcare professionals against acquired functional impairment. ${ }^{3,14}$

The salutogenic model of health describes the human tendency to adapt to acquired disability. This model predicts patient capacity to identify as healthy despite having medical pathology, and has been validated by systematic review. ${ }^{15,16}$ This capacity to identify as healthy is determined by three factors: the abilities to comprehend the illness, manage associated difficulties, and to find meaning in managing associated difficulties. The subjective worth of life and health is highly individualized as it is based upon the interaction of intrinsic patient factors, disease, and environment. The discrepancy between healthcare worker and patient evaluation of quality of life may be explained by healthcare workers focusing solely on relative functional loss rather than utilizing a salutogenic approach. ${ }^{12,14}$ Therefor, discrepancy between healthcare worker and patient evaluation of quality of life provides basis for deemphasis of healthcare worker opinion and increased emphasis on lived experience from patients in assessing quality of life. ${ }^{16}$ However, the question remains if this concept is applicable to neurosurgical patients.

A retrospective analysis demonstrated 11/13 severely disabled decompressive hemicraniectomy patients or their family members would consent the decompressive hemicraniectomy if given the option again. ${ }^{11}$ However, an 18-month follow-up of severely disabled or vegetative patients and their families demonstrated 10/19 families would provide consent knowing the outcome, but believed only $6 / 19$ patients would do the same. ${ }^{6}$ In another study, $82 \%$ of decompressive hemicraniectomy patients would have provided consent. ${ }^{17}$ However, the two most significantly impaired patients able to participate in the study would not have provided consent. In more extreme cases, not only can minimally conscious and persistently vegetative patients regain consciousness, ${ }^{18}$ but anecdotal experience of persistent near-vegetative state also demonstrates that some patients find worth in living after initial anguish. ${ }^{19,20}$ Even severely impaired neurosurgical patients commonly adapt, as expected by the salutogenic model of health. ${ }^{15}$ Due to previously described bias and lack of salutogenic approach, ${ }^{16}$ the neurosurgical team cannot accurately pass judgements regarding patient quality of life nor fitness to live. Therefor it is unethical to allow these judgements to impact decision to operate and another perspective for appraising the decision to operate should be sought.

While the majority of patients and substitute-decision makers have voiced patient desire to continue living paired with a high perceived quality of life, $6,11,17,20$ there remains a portion of patients whom are unable to voice their values. The true proportion of those who would prefer to die is not known given the small sample size of existing evidence and inability to communicate directly with the severely impaired. Therefor, the population of those who might choose death could be significantly higher. To avoid creating significant suffering, it is necessary to assume there is a sizeable population of those with acquired severe functional disability that would rather not live due to low perceived quality of life. 
Considering the conflict between voiced patient desire to live and assumed desire to die, an issue regarding treatment is presented. To deny the majority of cases would consign those who would want to live to death. To accept the majority of cases would consign those who wish to die to suffer. Furthermore, the proportions of these populations are unknown. An impasse is reached regarding the decision to operate as patient preference cannot be known until after surgery is performed.

The fundamental issue is that an unidentified patient's values or sociocultural beliefs cannot be known to infer best treatment. As discussed above, the healthcare team cannot impose sociocultural beliefs due to healthcare bias. The medical team cannot know the patient's salutogenic capacity for adaptation in order to predict perceived quality of life without further patient information. Given more time, it is possible to contact those who know the patient to gather critical information. Therefore, performing lifesaving surgery allows time to gather information, allowing an ethical solution.

After the patient had been operated on and presumably survived, further information regarding the patient would ideally obviate the patient's values and salutogenic capacity — whether the patient would accept or adapt to accept impaired survival. In the situation survival is within the patient's values or the patient is likely to adapt, the quandary was ethically navigated and beneficence was done. However, in the case survival is not within the patient's values nor is the patient likely to adapt, the quandary was ethically navigated but harm was done. By assessing salutogenic capacity, the team may ethically identify the ultimate outcome of the surgery, allowing further decision-making.

In the situation the surgery resulted in an undesirable outcome, further action is necessary. To rectify this, amends must be made in accordance with patient values. This may vary from support to reimbursement, but where the patient falls on this spectrum practically depends upon the stakeholder's values, capabilities of the healthcare system, and relevant jurisdictional legal constraints. For these reasons, the act of ethical reconciliation is beyond the scope of this paper.

The concepts described above may also be applied to situations in which patients are identifiable and substitute decision makers are present. As previously described by the salutogenic model of health, the neurosurgical team may appraise a patient's capacity to adapt and achieve acceptance with severe functional impairment. The team may specifically assess the three core qualities of the salutogenic model of health: ability to comprehend the illness, manage associated difficulties, and to find meaning in managing associated difficulties. Assessing the degree to which these core features exist within the patient will allow estimation of the likely perceived quality of life or the degree of adaptability. By de-emphasizing neurosurgeon appraisal of post-surgical quality of life and emphasizing patient degree of adaptability, neurosurgeons may mitigate their biases in conversation with substitute decision makers or patients. In the context no patient information is available with severe pathology, lifesaving operation is advocated so salutogenic appraisal is possible. By attempting to instate a salutogenic approach, the dilemma is ethically navigated although some patients may be appraised to have a low perceived quality of life.

\section{APPROACH TO MANAGEMENT}

Within modern medicine, the patient perspective is paramount in determining whether the patient should live or die. ${ }^{21}$ Ideally, the patient would be able to voice his or her values, but this is unlikely given poor pre-operative status. The neurosurgical team must attempt to gather as much information as possible from those close to the patient and make an appraisal of the patient's likely perception of a poor functional outcome, or degree of adaptability (Figure). 


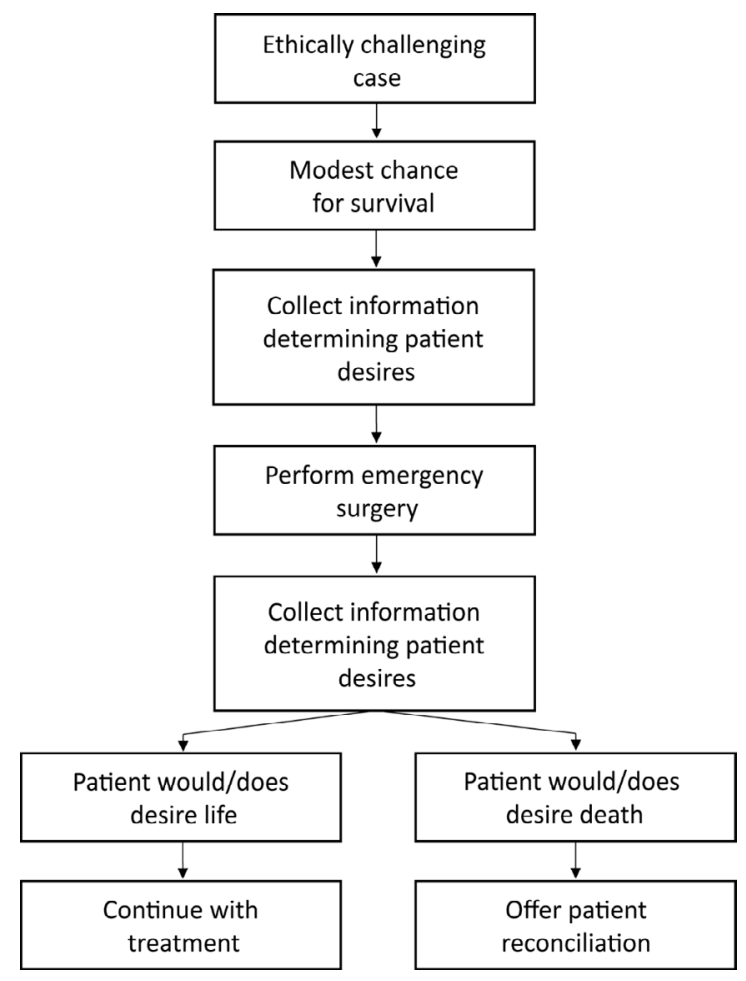

Fig. 1 Decision-making pathway for ethical management of severe emergency subdural hematoma unidentifiable patient.

This appraisal may utilize the salutogenic model of health to help determine whether or not to operate. However, if the patient is unidentifiable and there is no further time for information gathering, threshold for operation should be low despite poor functional outcome. By preferentially operating on the unidentified patient, two possible outcomes allow determination of the patient's quality of life post-surgery. The first possibility is that the patient can communicate his or her perspective of outcome. The second possibility is that the patient cannot communicate, and further time has been created to identify those close with the patient to assess the patient's likely outcome perspective or salutogenic capacity. Within this approach, an ethical decision is achievable.

Assuming the patient has survived and exists in a state of permanent functional deficit, action is required depending upon patient perceived quality of life. If the patient's outcome is perceived as acceptable, no further work is required as the ethical positive has been reached. If the patient's outcome is not perceived as acceptable, further work is required. Capacity for patient adaptation may be appraised within the context of the salutogenic model of health. ${ }^{15}$ Salutogenic appraisal of patient's capacity to adapt will allow determination of two things. First: if the patient's likely perspective is shared with those speaking on the patient's behalf. Second: if the patient may require more time or support to adapt to his or her outcome. If the patient is deemed to not have requisite capacity to achieve approval of functional outcome, reconciliation is required as a negative situation has been reached, albeit ethically. However, the nature of this reconciliation depends upon the patient, medical system, and legal system.

\section{CONCLUSION}

The judgement of quality of life is highly personal and there is strong evidence patients adapt to acquired disability. The healthcare team cannot make assumptions regarding patient post-operative quality of life without a salutogenic appraisal. Furthermore, if a patient is unidentifiable, the healthcare team must attempt to preserve life so that more 
information can be collected from those familiar with the patient, even if likely the outcome is severely functionally impaired. By utilizing this information within a salutogenic approach, a final decision regarding patient perceived quality of life is achievable. If harm has been done as the patient achieved a poor perceived outcome, reconciliation is required. This allows the problem of operating in a severe neurotrauma such as acute subdural hematoma to be navigated ethically.

\section{REFERENCES}

1. Weil A, Rahme R, Moumadjian R, Bouthillier A, Bojanowski M. Quality of life following hemicraniectomy for malignant MCA territory infarction. Can J Neurol Sci. 2011;38(3):434-438.

2. Honeybul S, Gillett G, Ho K. Futility in Neurosurgery: A Patient-Centered Approach. Neurosurgery. 2013;73(6):917-922. doi:10.1227/NEU.0000000000000014

3. Honeybul S, Kwok HM, Blacker DW. ORACLE Stroke Study: Opinion Regarding Acceptable Outcome Following Decompressive Hemicraniectomy for Ischemic Stroke. Neurosurgery. 2016;79(2):231-236. doi:10.1227/ NEU.0000000000001115

4. Marsh H. Do No Harm: Stories of Life, Death, and Brain Surgery.; 2016.

5. Honeybul S, Janzen C, Kruger K, Ho K. Decompressive craniectomy for severe traumatic brain injury: is life worth living? J Neurosurg. 2013;119(December):1566-1575.

6. Loew F. Ethics in Neurosurgery. Acta Neurochir (Wien). 1992;116:187-189.

7. Servadei F, Nasi M, Giuliani G, et al. CT prognostic factors in acute subdural haematomas: the value of the 'worst' CT scan. Br J Neurosurg. 2000;14(2):110-116.

8. Massaro F, Lanotte M, Faccani G, Triolo C. One hundred and twenty-seven cases of acute subdural haematoma operated on. Correlation between CT scan findings and outcome. Acta Neurochir (Wien). 1996;138(2):185-191.

9. Kameyama M, Karibe H, Onuma T, Tominaga T. Epidemiological study of head injury in Miyagi neurotrauma databank: age, cause of injury, pathophysiology and outcome. Neurotraumatology. 2008;31(1):49-56.

10. Nakamura H. Severe traumatic brain injury with acute subdural hematoma: clinical and pathophysiological aspect. Neurotraumatology. 2002;25:182-188.

11. Honeybul S, Gillett GR, Ho KM, Janzen C, Kruger K. Is life worth living? Decompressive craniectomy and the disability paradox. J Neurosurg. 2016;125(September):775-778. doi:10.3171/2016.3.JNS1683.

12. Lacey H, Fagerlin A, Loewenstein G, Smith D, Riis Ja, Ubel P. Are they really that happy Exploring scale recalibration in estimates of well-being. Heal Psychol. 2013;27(6):669-675. doi:10.1037/0278-6133.27.6.669. Are

13. Honeybul S, Ho K, Hanlon SO. Access to Reliable Information about Long-Term Prognosis Influences Clinical Opinion on Use of Lifesaving Intervention. PLoS One. 2012;7(2):1-7. doi:10.1371/journal.pone.0032375

14. Ubel P, Loewensein G, Schwarz N, Smith D. Misimagining the unimaginable: the disability paradox and health care decision making. Heal Psychol. 2005;24(4):57-62.

15. Antonosky A. The structure and properties of the sense of coherence scale. Soc Sci Med. 1993;36(6):725-733.

16. Eriksson M, Lindström B. Validity of Antonovsky's sense of coherence scale: A systematic review. J Epidemiol Community Health. 2005;59(6):460-466. doi:10.1136/jech.2003.018085

17. Dworkin R. Life's Dominion. An Argument about Abortion and Euthanasia. New York: Alfred A. Knopf Inc.; 1993. 
18. Kiphuth I, Kohrmann M, Lichy C, Schwab S, Huttner HB. Hemicraniectomy for Malignant Middle Cerebral Artery Infarction: Retrospective Consent to Decompressive Surgery Depends on Functional Long-Term Outcome. Neurocritical Care Soc. 2010;13:380-384. doi:10.1007/s12028-010-9449-8

19. Owen A. Into the Gray Zone A Neuroscientist Explores the Border Between Life and Death. Scribner; 2017.

20. Faugeras F, Rohaut B, Valente M, et al. Survival and consciousness recovery are better in the minimally conscious state than in the vegetative state. Brain Inj. 2018;32(1):72-77. doi:10.1080/02699052.2017.1364421

21. Andrews K. Clinical approaches to the withdrawal of nutrition and hydration. Clin Med (Northfield Il). 2003;3(4):342-345.

Citation: Calvin W. Howard BSc (Hon,), MD, "Ethical Discussion and Approach for the Disabling Acute Subdural Hematoma". American Research Journal of Clinical Case Reports, vol 2, no. 1, 2020, pp. 1-7.

Copyright (C) 2020 Calvin W. Howard BSc (Hon,), MD. This is an open access article distributed under the Creative Commons Attribution License, which permits unrestricted use, distribution, and reproduction in any medium, provided the original work is properly cited. 